\title{
A System Optimal Speed Advisory Framework for a Network of Connected and Autonomous Vehicles
}

\author{
Cuong H. P. Nguyen ${ }^{\circledR}$, Nam H. Hoang, Seunghyeon Lee, and Hai L. Vu
}

\begin{abstract}
The technological advancements involving information and communication technologies (ICT), such as Connected and Automated Vehicles (CAVs) and the Intelligent Transport Systems (ITS), have enabled new efficient traffic control and management strategies to mitigate traffic congestion. Specifically, the combined traffic flow-speed advisory systems based on CAVs and ITS technologies could provide the individual vehicle with the optimal speed to reduce the fuel consumption, the number of stops, simultaneously reduce the network-wide traffic congestion and improve road safety. This article develops a novel bilevel control framework underpinned by the mutual interaction between a system optimal traffic flow control strategy at a network level and a speed control policy for an individual vehicle at a link level within a connected traffic environment. Our framework proposes the novel group-based method to guarantee the consistency and interaction between the macroscopic and microscopic models. To this end, it efficiently optimizes vehicular trajectories while meeting the network-wide objectives which have not been investigated previously in the literature. We propose an efficient algorithm for this problem that iteratively solves mixed-integer linear programming (MILP) models for each upper and lower level. Numerical results indicate the effectiveness of the proposed speed advisory method in vehicular emission reduction, favorable network queue formation, and its positive influence on traffic flow patterns over the network.
\end{abstract}

Index Terms-Mixed-integer linear programming, dynamic traffic assignment, optimal speed advisory, bi-level control strategy, car following model, lane-changing model, connected and autonomous vehicles.

\section{INTRODUCTION}

$\mathbf{T}$ RAFFIC congestion is still a major problem in most metropolitan areas around the world, which has a significant negative impact on our society, environment, and economy. According to the report from the Bureau of Infrastructure, Transport and Regional Economics [1] in Australia, the congestion cost was estimated to be A $\$ 16.3$ billion in 2015 and predicted to grow by $5.5 \%$ per year from 2016 to 2030 .

A traditional solution to mitigate traffic congestion is to further build the transport infrastructure in order to increase

Manuscript received August 6, 2019; revised March 3, 2020, June 1, 2020, and November 17, 2020; accepted January 15, 2021. This work was supported by the Australian Research Council (ARC) Discovery Projects under Grant DP180102551. The Associate Editor for this article was Y. Wang. (Corresponding authors: Cuong H. P. Nguyen; Hai L. Vu.)

Cuong H. P. Nguyen, Nam H. Hoang, and Hai L. Vu are with the Monash Institute of Transport Studies, Department of Civil Engineering, Faculty of Engineering, Monash University, Melbourne, VIC 3800, Australia (e-mail: phucuong.nguyen@monash.edu; hai.vu@monash.edu).

Seunghyeon Lee is with the Faculty of Engineering and Information Technology, University of Technology Sydney, Ultimo, NSW 2007, Australia.

Digital Object Identifier 10.1109/TITS.2021.3056696 the network capacity which comes with significant costs not counting for major disruptions due to the spatial and temporal restrictions during the construction. More recently, the rapid technological advancements involving information and communication technologies (ICT), such as Connected and Automated Vehicles (CAVs) and the Intelligent Transport Systems (ITS), have opened up opportunities to facilitate new solutions for this rather old problem. In this article, we aim to develop new vehicular control and management strategies on a network of CAVs to reduce congestion and improve networkwide traffic efficiency.

Specifically, CAVs' speed can be controlled (via optimal speed advisory systems) to reduce the number of stops and redundant maneuvers in acceleration or deceleration, which eventually reduce traffic congestion and fuel consumption as well as improve network performance. Below we review the state-of-the-art speed advisory systems in the literature, and state our new contributions for this emerging area.

In the literature, existing speed advisory strategies can be broadly classified into two main categories: link speed advisory (LSA) and vehicle speed advisory (VSA).

Link speed advisory: LSA enforces or suggests the maximum speed for vehicles at a particular link or segment in a network through the help of roadside units or message signs. The displayed speed on these traffic signs is either static or dynamic depending on the corresponding control strategy.

The static speed limit (SSL) control problem is usually linked with the network design problem. [2] showed that imposing speed limits cannot always reduce the total travel time in a transportation network. [3] proposed a multiobjective bi-level programming model to design optimal link-specific speed limits that minimize travel time over the network, the number of expected accidents, and traffic exhaust emissions simultaneously in some predefined scenarios. This study showed that traffic network performance can be improved by imposing appropriate speed limits and there would be a trade-off among traffic safety, vehicular congestion, and fuel emissions. [4] investigated the effect of SSL control on the reallocation of equilibrium traffic flow where the result indicates that an appropriate speed control strategy can possibly emulate a road pricing scheme managing traffic flows in a network. Nevertheless, it is worth noting that the SSL control strategies have minimal or no impact on the mitigation of on-going congestion where vehicle's traveling speed is often far below the speed limit and fluctuates rapidly.

The dynamic speed limit (or variable speed limit - VSL) control method overcomes the SSL limitation by adapting the 
speed limit based on real-time traffic information. Approaches under this scheme can be further categorized into two main categories: a reactive and proactive approach. In the reactive approach, a detection of queue formation from real-time traffic information is a trigger to activate a controller for an interception at the following time interval. This approach has been studied both theoretically [5] and practically [6], where it shows the efficiency in improving traffic safety and mobility under congested conditions. However, its main limitation is the considerable time delay between the occurrence of traffic congestion and the corresponding control action.

In contrast, a proactive approach allows a controller to proactively respond to traffic conditions by using a predictive model of traffic flows. Herein, the formation of the vehicular queues can be anticipated in advance by a predictive model that provides useful input data to the controller and enables it to construct optimized control strategies in order to resolve a potential traffic breakdown. Model predictive control (MPC) is a common approach to implement proactive strategies where their performance is shown to improve throughput, safety, equity, and driver acceptance in scenarios involving free-way or work-zone areas [7], [8].

Despite the potential of a network-wide optimization, most existing studies however have not considered VSL in the context of a whole transportation network. Exceptions include [9] where the authors proposed a VSL control method based on a complex second-order traffic flow model for a ring-motorway network. Furthermore, [10] proposed a localized optimization of VSL for each road based on a Markov process where its state is obtained from a link transmission model (LTM). [11] reformulated this problem as a nonlinear mathematical program based on a cell transmission model. Nevertheless, all these LSA studies did not incorporate a speed control of an individual vehicle which would now be possible due to the emerging of CAVs.

Vehicle speed advisory: The existence of vehicle-to-vehicle $(\mathrm{V} 2 \mathrm{~V})$ and vehicle-to-infrastructure (V2I) communications enables CAVs to seamlessly connect to the surrounding vehicles in real-time as well as carry out their own driving maneuvers.

In this context, the VSA system would provide the optimal speed profile for each vehicle that aims to reduce a traffic accident, improve the efficiency of traffic flow, reduce fuel emissions at a signalized intersection. In literature, there has been a large body of works focusing on two problems of optimization: (1) a single-vehicle speed profile and (2) a multivehicle speed profile based on given objectives and constraints.

In the first problem, the algorithms generally provide the optimal speed profile that is only available to a designated vehicle. To this end, they utilize upcoming traffic signal information at the downstream signalized intersection and other approaching CAVs' profile for optimizing individual CAV's operation. These works are usually known as Optimal Speed Advisory (OSA) and/or Green Light Optimal Speed Advisory (GLOSA) systems that aim to minimize the stoppage time and emission/fuel consumption of vehicles approaching the intersection. [12] was the first to introduce the term GLOSA and its impact on a traffic network under diverse market penetration rates of connected vehicles. [13] further investigated the impact of GLOSA for autonomous vehicles (AVs). [14] proposed a framework to produce optimal speed profiles for individual vehicles traveling across a signalized arterial with consideration of queue lengths. [15] developed an algorithm for optimizing the delay over the entire trajectory of vehicles and proposed a time-loop technique for platoon-based optimization.

For environmental benefits, several similar studies in terms of eco-driving have been proposed. [16] introduced the approach of multi-stage dynamic programming method to efficiently minimize the total fuel emissions of vehicles by applying car following model on a platoon-based algorithm. [17] applied the augmented Lagrangian genetic algorithm (ALGA) on searching the optimal speed to minimize the fuel consumption and running time. They used a car-following model to guarantee the collision-free among vehicles. [18] introduced an eco-cooperative adaptive cruise control (EcoCACC) algorithm that seeks for a fuel-optimum vehicle trajectory while considers vehicular queue lengths at the intersection.

In contrast, existing methods for a multi-vehicle control scheme find an optimal solution derived from a joint optimization of multiple vehicles. [19] proposed an eco-driving system at an isolated signalized intersection under a partially connected traffic environment. [20] and [21] proposed a parsimonious shooting heuristic algorithm to smooth CAVs' trajectories approaching a signalized intersection while complying with constraints for vehicular arrivals, mechanical limits, traffic lights and safety of following vehicles. Similarly, [22] introduced an algorithm for the integrated optimization scheme that can simultaneously optimize the trajectories of CAVs and intersection controllers (i.e., signal timing and phasing of traffic signals). [23] presented a set of integer programming and dynamic programming models for scheduling longitudinal trajectories of CAVs that consider both systemwide safety and throughput requirements. [24] proposed a framework that provides optimal speed profiles to vehicles approaching bottlenecks in order to prevent traffic breakdowns. Despite considering multiple vehicles, none of the existing works has studied the effect of VSA in a networkwide context where coordinated vehicular movements along a signalized corridor and end-to-end vehicle routing decisions both play an important role in improving the system performance.

This article proposes a novel approach for the dynamic speed advisory (DSA) where we aim to (i) develop a network-wide speed advisory algorithm that is assumed to be deployed in every CAV inside the network, and (ii) evaluate the improvement in the network performance resulting from our proposed framework. Particularly, in tackling the second objective, we employ a range of approximations in our framework including the use of green time fractions (as discussed in Section II.B) and aggregation of flow (as discussed in Section II.C) to make our framework more scalable and less complex without compromising on the accuracy of our performance evaluation. To this end, the new contributions in this article are listed below: 
TABLE I

A List of Notations in the Paper

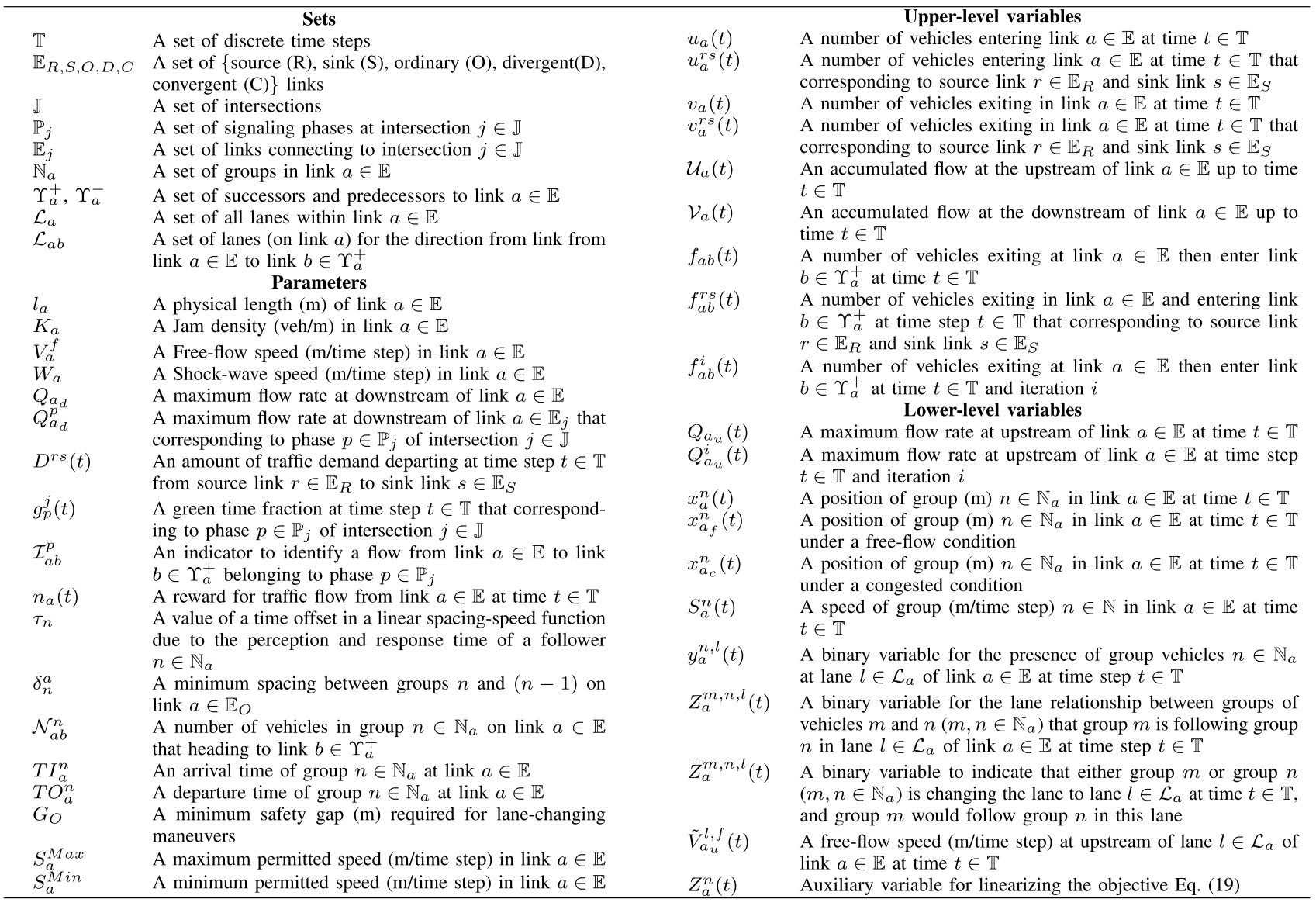

- a novel bi-level optimization control framework based on a system optimal dynamic traffic assignment with dynamic speed advisory (SODTA-DSA) that attains an optimal speed for each vehicle in a network;

- a group-based optimization model for speed harmonization in a multi-lane link where the longitudinal and lateral trajectories of groups are derived from the modified Newell's car following model (CFM);

- an efficient MILP method to solve the above framework; and showing the effectiveness of the proposed method in vehicular emission reduction, its impact on queuing formation, and also on the change of the traffic flow patterns over the whole network.

The rest of this article is organized as follows. Section II presents the formulation of a bi-level dynamic speed advisory framework. Section III introduces a solution method to effectively solve the designed formulation. The numerical results are shown in Section IV followed by conclusions in Section V.

\section{PRoblem Formulation}

In this section, we define a notation of variables and parameters, then present our bi-level mathematical framework for a SODTA-DSA problem.

Notation: The traffic network is considered as a directed graph $\mathcal{G}=(\mathbb{V}, \mathbb{E})$, which consists of a set of nodes $\mathbb{V}$ connected via a set of multi-lane links $\mathbb{E}$. Let $\mathbb{T}$ be a set of the discrete time used in this study. Let $\mathbb{E}_{R}, \mathbb{E}_{S}, \mathbb{E}_{O}, \mathbb{E}_{D}$, and $\mathbb{E}_{C}$ define a set of source links, sink links, ordinary links, divergent links, and convergent links, respectively. For each link $a \in \mathbb{E}$, let $\Upsilon_{a}^{+}, \Upsilon_{a}^{-}$represent a set of successors to link $a$, predecessors to link $a$ respectively. Let $\mathcal{L}_{a}$ and $\mathcal{L}_{a b}$ sequentially denote the set of all lanes and the set of lanes (on link $a$ ) for the direction from link $a$ to $b$. Besides, each link $a \in \mathbb{E}$ is also characterized by a set of parameters, including length $l_{a}$, jam density $K_{a}$, shock-wave speed $W_{a}$, free-flow speed $V_{a}^{f}$ and maximum flow rate at downstream $Q_{a_{d}}$. Decision variables are described appropriately in the following sections. Table I shows all notations where their descriptions are provided in relevant sections in this article.

Assumptions: In this section, we set the following assumptions for the development of our formulation:

- All vehicles are CAVs with negligible delay in the communication and response time.

- Signal timing is fixed with a given green split for each phase at each intersection within a constructed network.

- There is no over-taking between vehicles.

The first assumption enables all vehicles to receive and act on the updated instruction of routing and a speed control in real time. The remaining assumptions are obliged to develop the formulation that combines dynamic traffic 


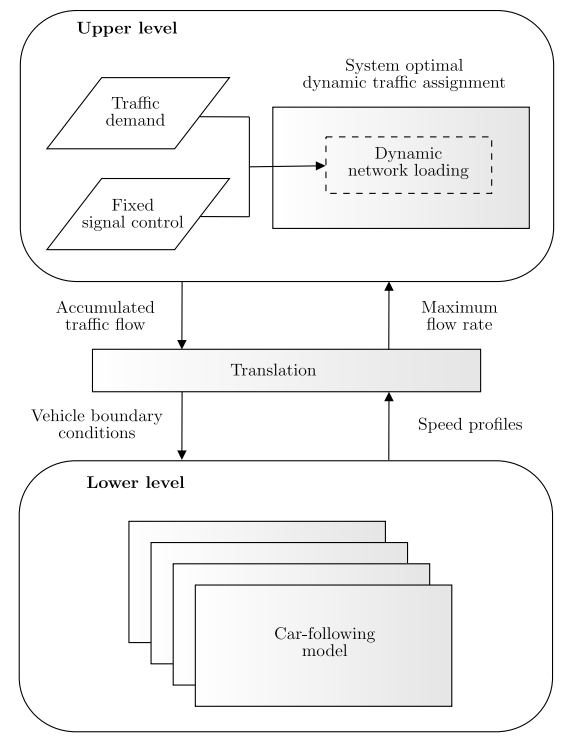

Fig. 1. Bi-level model structure.

assignment and car following models. Note that, the last assumption is to guarantee the first-in-first-out (FIFO) principle of vehicles in any part of the network, which is consistent with the assumptions of the dynamic network loading and Newell's CFM.

\section{A. Model Development}

Our proposed bi-level framework is demonstrated in Fig. 1. This article formulates the DSA problem as a bi-level formulation with special attention given to the modeling of CAVs' speed profiles from a microscopic perspective while taking into account the network-wide impacts of DSA in a macroscopic model at the same time. In this framework, the system optimal dynamic assignment is formulated at the upper level to minimize the total travel time of all CAVs in the network. It feeds the lower-level model with link flows which could be interpreted as the arrival, departure schedule, and the direction of vehicle movements. At the lower level (or the link level), we propose a speed harmonization model that optimizes the speed profile of CAVs where their trajectories are calculated from a group-based car-following model including lane-changing behavior. Note that, each link is treated separately therefore the complexity of the lowerlevel model is independent of the network scale. The lower model returns the feedback of CAVs' speed profiles regarding the constrained maximum flow rate at the upper level. The following sections present the details of their formulation.

\section{B. The Upper Level}

The formulation of the upper level is an extension of SODTA model in the work of [25] where we refer to this work for further comprehensive descriptions. Besides, we also consider a fixed signal timing scheme with given green splits into our formulation [26]. Note that, the maximum flow rate at upstream of the link $Q_{a_{u}}(t)$ becomes the variable in our work where it is derived from the lower level instead of assuming as a constant value as stated in the previous works. In summary, we use following variables at the upper-level:

- $u_{a}(t)$ : the number of vehicles entering link $a$ at time $t$.

- $u_{a}^{r s}(t)$ : the number of vehicles entering link $a$ at time $t$ that corresponding to source $r \in \mathbb{E}_{R}$ and sink $s \in \mathbb{E}_{S}$.

- $v_{a}(t)$ : the number of vehicles exiting in link $a$ at time $t$.

- $v_{a}^{r s}(t)$ : the number of vehicles exiting in link $a$ at time $t$ that corresponding to source $r \in \mathbb{E}_{R}$ and sink $s \in \mathbb{E}_{S}$.

- $f_{a b}(t)$ : the number of vehicles exiting in link $a$ and entering link $b$ at time $t$.

- $f_{a b}^{r s}(t)$ : the number of vehicles exiting in link $a$ and entering link $b$ at time $t$, corresponding to source $r \in \mathbb{E}_{R}$ and sink $s \in \mathbb{E}_{S}$.

- $Q_{a_{u}}(t)$ : the maximum flow rate at upstream of link $a$ at time $t$.

a) Objective function: The objective of SODTA is to minimize the total travel time of all CAVs which is equivalent to maximize throughput of transportation network [27].

$$
\max F=\left[\sum_{a \in \mathbb{E}_{S}} \sum_{t \in \mathbb{T}} \sum_{k=0}^{t} u_{a}(k)\right] .
$$

b) The link transmission-based constraints:

- A constraint for demand satisfaction:

$$
u_{r}^{r s}(t)=D^{r s}(t) \quad \forall t \in \mathbb{T}, r \in \mathbb{E}_{R}, s \in \mathbb{E}_{S}
$$

where $D^{r s}(t)$ denotes an amount of traffic demand departing at time $t \in \mathbb{T}$ from source $r \in \mathbb{E}_{R}$ to sink $s \in \mathbb{E}_{S}$.

- Link constraints:

- Incoming flow: $\forall t \in \mathbb{T}, \forall a \in \mathbb{E} \backslash \mathbb{E}_{R}$

$$
\begin{aligned}
& u_{a}(t) \leq K_{a} l_{a}+\sum_{k=0}^{t-\frac{l_{a}}{W_{a}}} v_{a}(k)-\sum_{k=0}^{t-1} u_{a}(k) \\
& u_{a}(t)=\sum_{r \in \mathbb{E}_{R}} \sum_{s \in \mathbb{E}_{S}} u_{a}^{r s}(t) \leq Q_{a_{u}}(t) .
\end{aligned}
$$

- Outgoing flow: $\forall t \in \mathbb{T}, \forall a \in \mathbb{E} \backslash \mathbb{E}_{S}$

$$
\begin{aligned}
& v_{a}^{r s}(t) \leq \sum_{k \in[0, t]: \frac{l a}{V_{a}^{f}}+k \leq t} u_{a}^{r s}(k)-\sum_{k=0}^{t-1} v_{a}^{r s}(k) \\
& v_{a}(t)=\sum_{r \in \mathbb{E}_{R}} \sum_{s \in \mathbb{E}_{S}} v_{a}^{r s}(t) \leq Q_{a_{d}} .
\end{aligned}
$$

- Node constraints:

- Traffic flow between connected links: $\forall t \in \mathbb{T}, \forall a \in$ $\mathbb{E} \backslash \mathbb{E}_{S}, \forall b \in \Upsilon_{a}^{+}, \forall r \in \mathbb{E}_{R}, \forall s \in \mathbb{E}_{S}$

$$
\begin{aligned}
& f_{a b}^{r s}(t) \geq 0 \\
& f_{a b}(t)=\sum_{r \in \mathbb{E}_{R}} \sum_{s \in \mathbb{E}_{S}} f_{a b}^{r s}(t) \\
& f_{a b}(t) \leq \frac{\left|\mathcal{L}_{a b}\right|}{\left|\mathcal{L}_{a}\right|} Q_{a_{d}}
\end{aligned}
$$

- Incoming flow: $\forall t \in \mathbb{T}, a \in \mathbb{E} \backslash \mathbb{E}_{R}, r \in \mathbb{E}_{R}, s \in \mathbb{E}_{S}$

$$
u_{a}^{r s}(t)=\sum_{b \in \Upsilon_{a}^{-}} f_{b a}^{r s}(t) .
$$


- Outgoing flow: $\forall t \in \mathbb{T}, a \in \mathbb{E} \backslash \mathbb{E}_{S}, r \in \mathbb{E}_{R}, s \in \mathbb{E}_{S}$

$$
v_{a}^{r s}(t)=\sum_{b \in \Upsilon_{a}^{+}} f_{a b}^{r s}(t) .
$$

c) The constraints for fixed signal control scheme: Let $\mathbb{J}$ denote the set of all intersections within the constructed network. For each intersection $j \in \mathbb{J}$, let us define $\mathbb{P}_{j}$ and $\mathbb{E}_{j}$ as the set of signaling phases at intersection $j$ and the set of links that connected to the intersection $j$, respectively. Then $g_{p}^{j}(t)$ indicates the green time fraction at time step $t \in \mathbb{T}$ that corresponding to phase $p \in \mathbb{P}_{j}$ of intersection $j \in \mathbb{J}$. To identify a flow from link $a \in \mathbb{E}$ to link $b \in \Upsilon_{a}^{+}$belonging to phase $p \in \mathbb{P}_{j}$, we use the indicator $\mathcal{I}_{a b}^{p}$, where

$$
\mathcal{I}_{a b}^{p}= \begin{cases}1 & \text { if the flow } a \rightarrow b \text { belongs to phase } p \\ 0 & \text { otherwise. }\end{cases}
$$

To this end, at each intersection $j \in \mathbb{J}$, the traffic flow exiting link $a \in \mathbb{E}_{j}$ at phase $p \in \mathbb{P}_{j}$ is constrained as below:

$$
\sum_{b \in \Upsilon_{a}^{+}} \mathcal{I}_{a b}^{p} f_{a b}(t) \leq g_{p}^{j}(t) Q_{a_{d}}^{p} .
$$

where $Q_{a_{d}}^{p}$ is a parameter that represents the maximum flow rate at downstream of link $a \in \mathbb{E}_{j}$ that corresponding to phase $p \in \mathbb{P}_{j}$ of intersection $j \in \mathbb{J}$.

Remark 1: Note that, the definition of $g_{p}^{j}(t)$ as a green time fraction is suitable for modeling the signal timings at large time intervals (covering single or multiple signal cycles per interval). It can be extended to include the detailed description of phase sequences or phase shifting by redefining this parameter at a smaller time interval (i.e., in a fraction of the signal cycle) as follows:

$$
g_{p}^{j}(t)= \begin{cases}1 & \text { if phase } p \text { is active at time step } t \\ 0 & \text { otherwise. }\end{cases}
$$

All the variables and constraints of the framework remain the same. The extension provides a higher resolution of the solution with significantly increased the complexity due to the requirement of the smaller time intervals. Given that our focus in this article has been the development of a mathematical framework for macroscopic traffic planning in largescale networks with $\mathrm{CAVs}$, the current green time fraction approach provides the right balance between complexity and scalability, and will be used in generating our numerical results later.

\section{The Lower Level}

A group-based speed optimization is a key component at the lower level based on the simplified car-following model [28]. This model is based on the arrival, departure schedule and the direction of moving of vehicles optimized at the upper level and the permitted speed range/lane occupancy at any link from traffic authorities as their constraints.

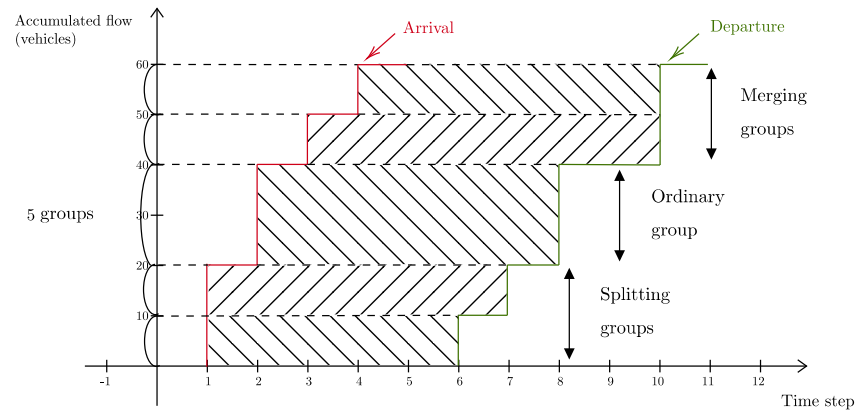

Fig. 2. Determination of groups in each link from the arrival and departure flows.

1) Translation of Macroscopic Output to the Input of Microscopic Model: We evaluate groups of vehicles in any link in the network based on their common arrival, departure time schedule and turning direction (more details are described in the following section) that obtained from the upper-level solutions, where the upper bound and lower bound are designated in Eqs. (4), and (6). In addition, our assumptions indicate that all vehicles are CAVs and the negligible delay in the communication and response time. Therefore, a vehicular group can be defined as a long vehicle, in which any personal vehicle within a group would share the same speed profile and behaviors.

a) Determination of groups: We determine a group formed at each link according to its arrival and departure flow as shown in Fig. 2. In other words, these groups of vehicles can be interpreted as an approximation for the aggregated traffic flows at the lower-level model. By considering a relationship between two consecutive groups, Fig. 2 shows three possible types of groups: splitting, ordinary and merging groups. Splitting groups enter a link at the same time step but leave the link at different time steps, meanwhile merging groups enter a link at different time steps however leave the link at the same time step. All groups that are not categorized as splitting or merging groups will be classified as ordinary groups. Note that, the splitting and merging among these groups of vehicles are only mathematical manipulations representing the aggregation of the microscopic flows in order to preserve the consistency of the assigned arrival and departure time schedule from the upper-level model. In practice, the proposed framework does not require vehicles to form a group (and thus group merging and splitting maneuver) at the link.

Let $\mathcal{U}_{a}(t)$ and $\mathcal{V}_{a}(t)$ define accumulated traffic flow at the upstream and downstream of link $a \in \mathbb{E}$ at time $t \in \mathbb{T}$, i.e.,

$$
\begin{aligned}
& \mathcal{U}_{a}(t)=\sum_{k \in \mathbb{T}: k \leq t} u_{a}(k) \\
& \mathcal{V}_{a}(t)=\sum_{k \in \mathbb{T}: k \leq t} v_{a}(k) .
\end{aligned}
$$

Let $\mathbb{N}_{a}$ define the set of all groups in the considered link $a \in \mathbb{E}$. Let $\mathcal{N}_{a b}^{m}$ denote the number of vehicles in group $m \in$ $\mathbb{N}_{a}$ on link $a \in \mathbb{E}$ that heading to link $b \in \Upsilon_{a}^{+}$. Then $\mathrm{TI}_{a}^{m}$ and $\mathrm{TO}_{a}^{m}$ are respectively defined as the arrival and departure time of group $m \in \mathbb{N}_{a}$ within link $a \in \mathbb{E}$. Besides, if link $a$ splits to $k$ links $\left\{b_{i}: i \in[1, k]\right\}$ at its downstream, for each direction 
from link $a$ to link $b_{i}$, we generate the groups of vehicles where the index $i$ (for the direction from $a$ to $b_{i}$ ) is encoded in the enumeration of group index as shown in Eqs. (16) to (18), as shown at the bottom of the page. Note that, the maximum size of a vehicular group is also bounded by $Q_{a_{u}}(t)$ where it is proportional to the time interval. Therefore, by using a small time interval, it also enables the study of interaction among individual vehicles at the lower model. We set up a dummy group where $T I_{a}^{0}=T O_{a}^{0}=0$, then apply the above formulation recursively to obtain all groups in any link.

Given these groups, the following sections present the model of speed harmonization based on Newell's CFM.

2) The Objective Function: Speed harmonization is one of the main approaches that could possibly reduce traffic congestion. In fact, it aims to suggest optimal speed for vehicles from far upstream of a bottleneck or an intersection to avoid queue spillovers and gridlocks, to minimize the idle time of vehicles and the number of vehicular stops. Besides the main benefit of smoothing movements of traffic flow, vehicles can also minimize fuel consumption.

In this work, we formulate a group-based speed harmonization by minimizing the variation of speed profile over time. Hence, the objective function at this level is expressed as below:

$$
S_{a}^{n}(t)=\underset{\forall a \in \mathbb{E}}{\operatorname{argmin}}\left[\sum_{\forall n \in \mathbb{N}_{a}} \sum_{\forall t \in \mathbb{T}}\left|S_{a}^{n}(t)-S_{a}^{n}(t-1)\right|\right]
$$

where $S_{a}^{n}(t)$ is the instantaneous speed of a group $n \in \mathbb{N}_{a}$ at a link $a \in \mathbb{E}$ and at a time step $t \in \mathbb{T}$. The above non-linear objective function Eq. (20) is linearized by using the auxiliary variables $Z_{a}^{n}(t)$ as follows:

$$
\begin{aligned}
& S_{a}^{n}(t)=\underset{\forall a \in \mathbb{E}}{\operatorname{argmin}}\left[\sum_{\forall n \in N_{a}} \sum_{\forall t \in T} Z_{a}^{n}(t)\right] \\
& Z_{a}^{n}(t) \geq S_{a}^{n}(t)-S_{a}^{n}(t-1) \quad \forall t \in \mathbb{T}, \quad \forall n \in \mathbb{N}_{a} \\
& Z_{a}^{n}(t) \geq S_{a}^{n}(t-1)-S_{a}^{n}(t) \quad \forall t \in \mathbb{T}, \quad \forall n \in \mathbb{N}_{a} .
\end{aligned}
$$

3) The Modified Group-Based Newell's Car Following Model: Let $x_{a}^{n}(t), x_{a_{f}}^{n}(t)$ and $x_{a_{c}}^{n}(t)$ present the position of group $n \in \mathbb{N}_{a}$, its position in a free-flow regime, and a congested regime within link $a \in \mathbb{E}$, respectively. Let $\tau_{n}$ represent the time offset in a linear spacing-speed function due to the perception and reaction time of a follower $n$ that follows the leader. In addition, $\delta_{n}^{a}$ describes the minimum space between the follower $n \in \mathbb{N}_{a}$ and the leader $(n-1) \in \mathbb{N}_{a}$ under a congested regime corresponding to link $a \in \mathbb{E}$. According to Newell's CFM, the position of group $n \in \mathbb{N}_{a}$ that follows its predecessor group $(n-1) \in \mathbb{N}_{a}$ at any time $t \in \mathbb{T}$ is determined by the minimum value between its position in a free-flow regime and a congested regime. Therefore, its mathematical expression is described below:

$$
\begin{aligned}
x_{a}^{n}(t) & =\min \left[x_{a_{f}}^{n}(t), x_{a_{c}}^{n}(t)\right] \quad \forall n \in \mathbb{N}_{a} \\
x_{a_{f}}^{n}(t) & =x_{a}^{n}\left(t-\tau_{n}\right)+V_{a}^{f} \tau_{n} \quad \forall n \in \mathbb{N}_{a} \\
x_{a_{c}}^{n}(t) & =x_{a}^{n-1}\left(t-\tau_{n}\right)-\delta_{n}^{a} \quad \forall n \in \mathbb{N}_{a} .
\end{aligned}
$$

Eq. (24) provides the location of group $n$ at the current time $t$ which is the location of the previous time plus the distance traversed with free-flow speed $V_{a}^{f}$ during the time difference $\tau_{n}$ between the current and previous time epoches. Eq. (26) is a discretized version of Eq. (24) where the $x_{a}^{n}(t)$ continuous function in Eq. (24) is discretized based on the system time unit. For free flow traffic conditions, the two equations are equivalent. Besides, $\tau_{n}$ is very small, hence we approximate $x_{a}^{n-1}\left(t-\tau_{n}\right)$ in Eq. (25) as $x_{a}^{n-1}(t)$ in Eq. (27). Therefore, the position of any group $n \in \mathbb{N}_{a}$ within link $a \in \mathbb{E}$ under a free-flow and a congested regime at Eqs. (24) and (25) can be modified while keeping the order of groups as below:

$$
\begin{gathered}
x_{a_{f}}^{n}(t)=x_{a}^{n}(t-1)+V_{a}^{f} \quad \forall t \in \mathbb{T}, \forall n \in \mathbb{N}_{a} \\
x_{a_{c}}^{n}(t)=x_{a}^{n-1}(t)-\delta_{n}^{a} \quad \forall t \in \mathbb{T}, \forall n \in \mathbb{N}_{a} .
\end{gathered}
$$

where the spacing $\delta_{n}^{a}$ between two consecutive groups $n \in \mathbb{N}_{a}$ and $(n-1) \in \mathbb{N}_{a}$ is also set to be proportional to the length of the group of followers as below:

$$
\delta_{n}^{a}=\mathcal{N}_{a b}^{n} * \frac{1}{K_{a}} .
$$

Remark 2: As the length $\delta_{n}^{a}$ should be smaller than the link length $l_{a}$, we choose the time interval such that:

$$
\delta_{n}^{a} \leq l_{a} \Leftrightarrow \mathcal{N}_{a b}^{n} \leq K_{a} l_{a} .
$$

Due to $\mathcal{N}_{a b}^{n} \leq Q_{a_{u}}(t)$, we can set the time interval such that the link flow capacity $Q_{a_{u}}(t)$ is bounded as:

$$
Q_{a_{u}}(t) \leq K_{a} l_{a} .
$$

Note that, Eq. (27) is slightly different from the original Eq. (25) to enable the merging and splitting scenario of groups as showed in Fig. 2. In more details, for any two successive groups $n$ and $(n-1)$ in $\mathbb{N}_{a}$, if they are categorised as merging groups, our model enables this behaviour by adding Eq. (30) to allow group $n$ and $(n-1)$ exit the link $a$ at the same time interval $T O_{a}^{n-1}=T O_{a}^{n}$.

$$
x_{a}^{n-1}\left(T O_{a}^{n-1}-1\right) \leq l_{a} \leq x_{a}^{n}\left(T O_{a}^{n}\right) \leq x_{a}^{n-1}\left(T O_{a}^{n-1}\right)-\delta_{n}^{a} .
$$

In contrast, under an splitting circumstance, the group $n \in$ $\mathbb{N}_{a}$ can be deferred by traversing at lower speed than the group

$$
\begin{aligned}
T I_{a}^{n k+i} & =T I_{a}^{n k}=\min _{t}\left[t: \mathcal{U}_{a}(t)>\min \left\{\mathcal{U}_{a}\left(T I_{a}^{(n-1) k}\right), \mathcal{V}_{a}\left(T O_{a}^{(n-1) k}\right)\right\}\right] \\
T O_{a}^{n k+i} & =T O_{a}^{n k}=\min _{t}\left[t: \mathcal{V}_{a}(t)>\min \left\{\mathcal{U}_{a}\left(T I_{a}^{(n-1) k}\right), \mathcal{V}_{a}\left(T O_{a}^{(n-1) k}\right)\right\}\right] \\
\mathcal{N}_{a b_{i}}^{n k+i} & =\frac{f_{a b_{i}}\left(T O_{a}^{n k}\right)}{v_{a}\left(T O_{a}^{n k}\right)}\left(\min \left\{\mathcal{U}_{a}\left(T I_{a}^{n k}\right), \mathcal{V}_{a}\left(T O_{a}^{n k}\right)\right\}-\min \left\{\mathcal{U}_{a}\left(T I_{a}^{(n-1) k}\right), \mathcal{V}_{a}\left(T O_{a}^{(n-1) k}\right)\right\}\right) .
\end{aligned}
$$


$(n-1) \in \mathbb{N}_{a}$ after time $T I_{a}^{n}=T I_{a}^{n-1}$ in order to finish its trip in link $a$ later than group $(n-1)$ although they enter the link at the same time. And hence, their trajectory should satisfy the following conditions:

$$
\begin{aligned}
x_{a}^{n-1}\left(T O_{a}^{n-1}-1\right) & \leq l_{a} \leq x_{a}^{n-1}\left(T O_{a}^{n-1}\right) \\
x_{a}^{n}\left(T O_{a}^{n}-1\right) & \leq l_{a} \leq x_{a}^{n}\left(T O_{a}^{n}\right)
\end{aligned}
$$

where $T O_{a}^{n}>T O_{a}^{n-1}$. Then we replace Eq. (24) and Eq. (25) to Eq. (23) and transform it into a linear form by relaxing to:

$$
\begin{aligned}
& x_{a}^{n}(t) \leq x_{a}^{n}(t-1)+V_{a}^{f} \quad \forall t \geq \mathrm{TI}_{a}^{n}+1, \forall n \in \mathbb{N}_{a} \\
& x_{a}^{n}(t) \leq x_{a}^{n-1}(t)-\delta_{n}^{a} \quad \forall t \geq \mathrm{TI}_{a}^{n}+1, \forall n \in \mathbb{N}_{a} .
\end{aligned}
$$

Additionally, the traveling speed of a group is derived from its trajectory as follow:

$$
S_{a}^{n}(t)=x_{a}^{n}(t+1)-x_{a}^{n}(t) .
$$

4) Lane-Changing Constraints: Groups of vehicles can perform lane-changing maneuvers while traveling along with the multi-lane link while satisfying the FIFO requirements. Let $y_{a}^{n, l}(t)$ be the binary variable to indicate the presence of group $n \in \mathbb{N}_{a}$ at lane $l \in \mathcal{L}_{a}$ of link $a \in \mathbb{E}$ at time step $t \in \mathbb{T}$. As each group of vehicles $n$ is only at one lane at any time, it means

$$
\sum_{\forall l \in \mathcal{L}_{a}} y_{a}^{n, l}(t)=1 \quad \forall t \in \mathbb{T}, \forall n \in \mathbb{N}_{a} .
$$

Besides, we also assume that each group $n$ can only change to the adjacent lanes in each time interval, hence it is formulated as:

$$
\sum_{\max (1, l-1)}^{\min \left(l+1,\left|\mathcal{L}_{a}\right|\right)} y_{a}^{n, l}(t) \geq y_{a}^{n, l}(t-1) \quad \forall t \in \mathbb{T}, \quad \forall n \in \mathbb{N}_{a} .
$$

The above constraint ensures that whenever group $n$ stays in lane $l$ at time $(t-1)$, then it can change to lane max $(1, l-1)$ or $\min \left(l+1,\left|\mathcal{L}_{a}\right|\right)$ at the next time step $t$. Let the binary variable $Z_{a}^{m, n, l}(t)$ indicate that group $m$ is following group $n$ in lane $l \in \mathcal{L}_{a}$ of link $a \in \mathbb{E}$ at time step $t \in \mathbb{T}$. The consistency between $Z$ and $y$ is presented by the following constraints:

$$
\begin{aligned}
Z_{a}^{m, n, l}(t)+Z_{a}^{n, m, l}(t) & \leq 1 \\
y_{a}^{m, l}(t)+y_{a}^{n, l}(t) & \leq 1+Z_{a}^{m, n, l}(t)+Z_{a}^{n, m, l}(t) \\
Z_{a}^{m, n, l}(t)+Z_{a}^{n, m, l}(t) & \leq y_{a}^{m, l}(t) \\
Z_{a}^{m, n, l}(t)+Z_{a}^{n, m, l}(t) & \leq y_{a}^{n, l}(t) .
\end{aligned}
$$

These constraints Eqs. (38) to (41) ensures that if groups $m$ and $n$ are on the same lane $l\left(y_{a}^{m, l}(t)=y_{a}^{n, l}(t)=1\right)$ at time $t$, then either $Z_{a}^{m, n, l}(t)$ or $Z_{a}^{n, m, l}(t)$ has to be 1 . In addition, when $m$ follows $n$ on lane $l$ at time $t$, the minimum spacing $\delta_{n}^{a}$ must also be satisfied, that means:

$$
M\left[Z_{a}^{m, n, l}(t)-1\right] \leq x_{a}^{n}(t)-x_{a}^{m}(t)-\delta_{n}^{a} .
$$

Moreover, when vehicles change their lanes, the safety gap acceptance $G_{0}$ is applied for any vehicle around the location of changing lane. Let $\bar{Z}_{a}^{m, n, l}(t)$ be a binary variable to indicate that either group $m$ or group $n$ is changing the lane to lane $l$ at time $t$, and group $m$ would follow group $n$ in this lane. Therefore,

$$
\begin{aligned}
& \bar{Z}_{a}^{m, n, l}(t) \geq Z_{a}^{m, n, l}(t)-Z_{a}^{m, n, l}(t-1) \\
& \bar{Z}_{a}^{m, n, l}(t) \geq Z_{a}^{m, n, l}(t-1)-Z_{a}^{m, n, l}(t) .
\end{aligned}
$$

And hence, the constraint for the safety gap acceptance $G_{0}$ is formulated as below:

$$
M\left(\bar{Z}_{a}^{m, n, l}(t)-1\right) \leq x_{a}^{n}(t)-x_{a}^{m}(t)-G_{0} .
$$

5) Boundary Constraints for Group: Time and space boundary conditions for an individual group are provided by the output from a macroscopic level and listed as below:

- Maintaining inflow and outflow of any link:

$$
\begin{aligned}
& x_{a}^{n}(t)=0 \quad \forall t \in \mathbb{T}: t \leq \mathrm{TI}_{a}^{n}, \forall n \in \mathbb{N}_{a} \\
& x_{a}^{n}(t) \geq l_{a} \quad \forall t \in \mathbb{T}: t \geq \mathrm{TO}_{a}^{n}, \forall n \in \mathbb{N}_{a} .
\end{aligned}
$$

- Exiting the link at the permitted lanes that corresponding to its direction of moving:

$$
\sum_{\forall l \in \mathcal{L}_{a b}} y_{a}^{n, l}(t)=1 \quad \forall t \in \mathbb{T}: t \geq \mathrm{TO}_{a}^{n}, \forall n \in \mathbb{N}_{a}
$$

- Abiding the permitted speed range from authorities:

$$
S_{a}^{M i n} \leq S_{a}^{n}(t) \leq S_{a}^{\text {Max }} \quad \forall t \in \mathbb{T}, \forall n \in \mathbb{N}_{a} .
$$

where $S_{a}^{\text {Min }}$ and $S_{a}^{\text {Max }}$ are the minimum speed and the maximum speed allowed on link $a \in \mathbb{E}$, respectively.

6) Feedback of Speed From the Lower Level to Upper Level: By controlling the speed trajectories of vehicles at the lower level, we influence the traffic flow entering a link at any time. From the upper-level's perspective, this effect is presented by the change of maximum flow rate at the upstream node of every link in the network. To capture this dynamic change, we measured the change of free-flow speed at the beginning of the multi-lane link for each lane at every time-step and update the new aggregated maximum flow rate at upstream $Q_{a_{u}}(t)$ to the upper level accordingly. Let $\tilde{V}_{a_{u}}^{l, f}(t)$ define this maximum speed among vehicles that enter lane $l \in \mathcal{L}_{a}$ of link $a \in \mathbb{E}$ at time step $t \in \mathbb{T}$, i.e.,

$$
\tilde{V}_{a_{u}}^{l, f}(t)=\max \left[S_{a}^{n}(t) \forall n \in \mathbb{N}: t=\operatorname{TI}_{a}^{n}, y_{a}^{n, l}(t)=1\right] .
$$

Therefore, the aggregated maximum flow rate at upstream $Q_{a_{u}}(t)$ is updated to the upper-level model regarding the triangular fundamental density-flow relation as follows:

$$
Q_{a_{u}}(t)=\sum_{\forall l \in \mathcal{L}_{a}} \frac{K_{a} \tilde{V}_{a_{u}}^{l, f}(t) W_{a}}{\left|\mathcal{L}_{a}\right|\left(\tilde{V}_{a_{u}}^{l, f}(t)+W_{a}\right)} .
$$

\section{Solution Method}

It is well-known that the bi-level programming problem is NP-hard [29]. Herein, we propose an efficient fixed point method to compute the optimal solution by repeatedly solving MILP problems at each level using the Gurobi solver [30]. The procedure of our method is described below:

- Step 1: Solve the non-holding-back SODTA model at the first iteration (further detail below in Section III.a). The obtained value of the SO objective, called $F^{*}$, 
is guaranteed in Step 4 to ensure the SO feasible domain while solving the optimal speed advisory.

- Step 2: Determine groups in each link as described in Eqs. (14)-(18).

- Step 3: In each link $a \in \mathbb{E}$, given the groups with their arrival and departure time, solve the lower-level model (for each link) to find optimal speed profiles: $\min _{S_{a}^{n}(t)}\left[\sum_{\forall n \in \mathbb{N}_{a}} \sum_{\forall t \in \mathbb{T}} Z_{a}^{n}(t)\right]$

s.t: $\quad$ Linear constraints of $Z_{a}^{n}(t)$ : Eqs. (21)-(22)

Microscopic car-following model: Eqs. (30)-(35)

Lane-changing behaviors: Eqs. (36)-(45)

Boundary condition for vehicles: Eqs. (46)-(49).

- Step 4: Given the CAVs' speed profiles from Step 3, update $Q_{a}^{u}(t)$ as mentioned in Eqs. (50) and (51) then solve the SODTA model (more detail described below in Section III.b).

- Step 5: The condition of convergence is presented below:

$$
\sum_{\forall a \in \mathbb{E}} \sum_{\forall b \in \Upsilon_{a}^{+}} \sum_{t \in \mathbb{T}}\left|f_{a b}^{i}(t)-f_{a b}^{i-1}(t)\right| \leq \varepsilon
$$

where $f_{a b}^{i}(t)$ is the value of $f_{a b}(t)$ in the iteration $i$. Besides, $\epsilon$ is equal to 0.01 in our numerical examples. If Eq. (52) is unsatisfied then return to step 2, otherwise return the solution and terminate the algorithm.

In the following part, we explain the details of the implementation in Steps 1 and 4 then discuss the uniqueness of Step 3 solutions.

\section{A. Non-Holding Back SODTA Solution in Step 1}

In the macroscopic model, in order to avoid solutions that hold back a portion of the traffic demand despite the available capacity downstream, we modified the original upper-level objective function Eq. (1) to a weighted function that preserves the SO solution while eliminating the holding back issue as follows:

$$
\max \sum_{\forall a \in \mathbb{E}_{S}} \sum_{t \in \mathbb{T}} \sum_{k=0}^{t} u_{a}(k)+m \sum_{\forall a \in \mathbb{E} \backslash \mathbb{E}_{S}} \sum_{\forall b \in \Upsilon_{a}^{+}} n_{a}(t) f_{a b}(t)
$$

where $n_{a}(t)$ represents a reward for the traffic flow from link $a \in \mathbb{E}$ at time step $t \in \mathbb{T}$, and it is designed to satisfy the condition: $n_{a}(t-1)>n_{a}(t)>0$. This reward helps to avoid the holding-back issue in the resulting solution [31]. The parameter $m$ is a very small number (i.e., $m=0.00001$ in our numerical results) to ensure that the SO objective is not significantly affected by this holding back cost. The full description of the SO DTA in Step 1 is summarized as below:

Objective: Eq. (53)

s.t: Macroscopic loading model: Eqs. (2) - (13).

\section{B. The SO DTA Model in Step 4}

[32] showed that the solution of the SODTA model is not unique where completely different traffic flow patterns could result in the same objective value. Therefore, after updating the link flow capacity $Q_{a_{u}}(t)$, we experienced the possibility of non-convergence of the solution where it might exist a loop of changes with corresponding flow patterns. For example:

$$
\begin{aligned}
Q_{a_{u}}^{i-1} \rightarrow f_{a b}^{i-1}(\text { Step } 1) & \rightarrow Q_{a_{u}}^{i}(\text { Step } 3) \rightarrow f_{a b}^{i}(\text { Step } 1) \\
& \rightarrow Q_{a_{u}}^{i+1}=Q_{a_{u}}^{i-1}(\text { Step } 3) \rightarrow \ldots
\end{aligned}
$$

where $Q_{a_{u}}^{i}$ and $f_{a b}^{i}$ are values of a link capacity and flow pattern at the iteration $i$, respectively. It shows that the solution at the iteration $(i+1)$ will be identical to the solution at the iteration $(i-1)$.

Besides, [33] also noticed this issue in a study of user equilibrium DTA and then, proposed a so-called condition of proportionality to study convergence in an optimization process. Similarly, we proposed a condition related to turning fractions at diverging and merging links to overcome such a problem in this work. Particularly, from the second iteration of our method, a modification to the upper-level framework has been proposed as follow:

- The objective of the upper-level model (Eq. (54)) in Step 4 is to minimize the variation of turning fractions at diverging and merging links. The absolute terms in Eq. (54) can be transformed into a linear form by introducing subsidiary variables $X_{b}^{c}(t), Y_{b}^{c}(t)$ and constraints as described in Eqs. (55) - (59).

- It is worth noting that we can guarantee the result of SO solutions (i.e., minimal total travel time) by maintaining the value of $F^{*}$ computed in Step 1. In addition, it takes into account the updated parameter of the maximum flow rate at upstream $Q_{a_{u}}(t)$ of link $a \in \mathbb{E}$. Hence, to fulfill this purpose, the below constraint is added to the original macroscopic model alongside the dynamic network loading model:

$$
\sum_{a \in \mathbb{E}_{S}} \sum_{t \in \mathbb{T}} \sum_{k=0}^{t} u_{a}(k)=F^{*} .
$$

In summary, the Step 4 model is formulated as below:

Objective : Eq.(55)

s.t : Macroscopic loading model: Eqs. (2) - (13)

Auxiliary constraints: Eqs. (56) - (59)

Domain of SO solutions: Eq. (60).

Remark 3: At Step 3, if the solution shows that each group of vehicles does not change their traveling speed during their travel in a particular link then the solution is unique for the SH problem at this link. In fact, with this condition, it means that for each group $n$ arriving link $a$ at time $T I_{a}^{n}$ and departing this link at time $T O_{a}^{n}$, if it runs at the same speed in link $a$, then its speed is equal to $S_{a}^{n}(t)=\frac{l_{a}}{T O_{a}^{n}-T I_{a}^{n}}$. Note that, $T I_{a}^{n}$ and $T O_{a}^{n}$ are the input to the model in this step.

Remark 4: The complexity in terms of the number of constraints and variables are described in Table III. In our numerical result, it shows that $\left|\mathbb{N}_{a}\right| \ll\left|\mathbb{E}_{R}\right|\left|\mathbb{E}_{S}\right||\mathbb{E}|$, therefore the computational effort for the lower model is significantly smaller than the upper model. 
TABLE II

LINEARIZATION OF THE OBJECTIVE EQ. (54) USED IN STEP 4

$\min \left\{\sum_{\forall a \in \mathbb{E}_{D}} \sum_{\forall b \in \Upsilon_{a}^{+}} \sum_{\forall c \in\left\{\Upsilon_{a}^{+} \backslash b\right\}} \sum_{\forall t \in \mathbb{T}}\left|u_{b}(t)-u_{c}(t)\right|+\sum_{\forall a \in \mathbb{E}_{C}} \sum_{\forall b \in \Upsilon_{a}^{-}} \sum_{\forall c \in\left\{\Upsilon_{a}^{-} \backslash b\right\}} \sum_{\forall t \in \mathbb{T}}\left|v_{b}(t)-v_{c}(t)\right|\right\}$

is transformed to

$$
\min \left\{\sum_{\forall a \in \mathbb{E}_{D}} \sum_{\forall b \in\left\{\Upsilon_{a}^{+} \backslash c\right\}} \sum_{\forall c \in\left\{\Upsilon_{a}^{+} \backslash b\right\}} \sum_{\forall t \in \mathbb{T}} X_{a}^{b c}(t)+\sum_{\forall a \in \mathbb{E}_{C}} \sum_{\forall b \in\left\{\Upsilon_{a}^{-} \backslash c\right\}} \sum_{\forall c \in\left\{\Upsilon_{a}^{-} \backslash b\right\}} \sum_{\forall t \in \mathbb{T}} Y_{a}^{b c}(t)\right\}
$$

where

$$
\begin{aligned}
X_{a}^{b c}(t) & \geq u_{b}(t)-u_{c}(t) \\
X_{a}^{b c}(t) & \geq u_{c}(t)-u_{b}(t) \\
Y_{a}^{b c} & \geq v_{b}(t)-v_{c}(t) \\
Y_{a}^{b c} & \geq v_{c}(t)-v_{b}(t)
\end{aligned}
$$

$$
\begin{aligned}
& \forall a \in \mathbb{E}_{D}, \forall b \in \Upsilon_{a}^{+}, \forall c \in\left\{\Upsilon_{a}^{+} \backslash c\right\}, \forall t \in \mathbb{T} \\
& \forall a \in \mathbb{E}_{D}, \forall b \in \Upsilon_{a}^{+}, \forall c \in\left\{\Upsilon_{a}^{+} \backslash c\right\}, \forall t \in \mathbb{T} \\
& \forall a \in \mathbb{E}_{C}, \forall b \in \Upsilon_{a}^{-}, \forall c \in\left\{\Upsilon_{a}^{-} \backslash c\right\}, \forall t \in \mathbb{T} \\
& \forall a \in \mathbb{E}_{C}, \forall b \in \Upsilon_{a}^{-}, \forall c \in\left\{\Upsilon_{a}^{-} \backslash c\right\}, \forall t \in \mathbb{T} .
\end{aligned}
$$

TABLE III

Complexity of Our Solution Method

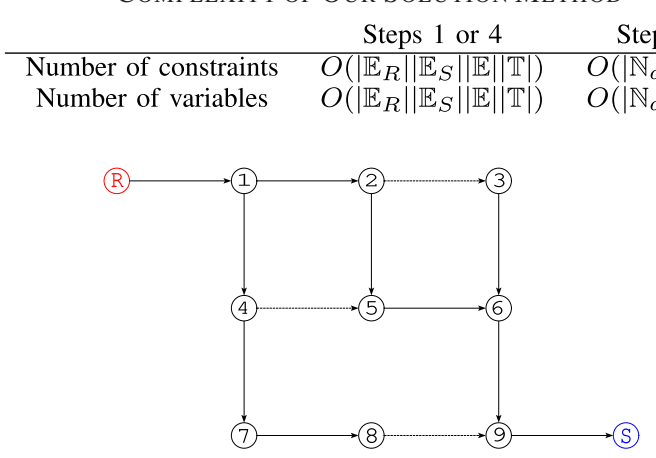

Fig. 3. Grid $3 \times 3$ network topology.

\section{Numerical Results}

In this section, we applied the proposed method to a variety of multi-lane transportation networks. For simplicity, the general rule for lane occupancy regarding the direction of movement at an intersection is inferred from the assumption that a through movement can take any lane of the link while a left or right turn movement is only allowed at the leftmost or rightmost lane of the link, respectively. In the small network, we aim to show the detailed information of the solution in terms of the harmonization of speed, flow swapping, queue formation, and also the comparison between local SH and network-wide SH control scheme. For the large network, we discuss the complexity of our method and evaluate the network-wide impact of VSA. Also in this section, the non-SH result is the solution obtained at Step 1 of our solution method, while the SH result is acquired after the convergence of the solution. Additionally, for the non-SH solution, all vehicles

\begin{tabular}{|c|c|c|}
\hline \multicolumn{3}{|c|}{ Traffic parameters } \\
\hline \multicolumn{3}{|c|}{ Applied to network } \\
\hline Time period & \multirow{3}{*}{\multicolumn{2}{|c|}{$\begin{array}{c}16.5 \mathrm{~min} \\
10 \mathrm{~s} \\
222 \mathrm{veh} / \mathrm{min} \text { in } 4.5 \mathrm{~min}\end{array}$}} \\
\hline Time-step duration & & \\
\hline Demand & & \\
\hline \multicolumn{3}{|c|}{ Applied to links } \\
\hline Parameters & Normal links & Bottleneck links \\
\hline Free-flow speed $\left(V_{f}^{a}\right)$ & \multicolumn{2}{|c|}{$20 \mathrm{~m} / \mathrm{s}$} \\
\hline Backward wave speed $\left(W_{a}\right)$ & \multicolumn{2}{|c|}{$10 \mathrm{~m} / \mathrm{s}$} \\
\hline Maximum permitted speed $\left(S_{a}^{M a x}\right)$ & \multicolumn{2}{|c|}{$20 \mathrm{~m} / \mathrm{s}$} \\
\hline Length of link $\left(l_{a}\right)$ & \multicolumn{2}{|c|}{$1600 \mathrm{~m}$} \\
\hline Total lane of link $\left(\mathcal{L}_{a}\right)$ & \multicolumn{2}{|c|}{2 lanes } \\
\hline Jam density $\left(K_{a}\right)$ & $0.3 \mathrm{veh} / \mathrm{m}$ & $0.075 \mathrm{veh} / \mathrm{m}$ \\
\hline Maximum flow capacity $\left[Q_{a_{u}}(t), Q_{a_{d}}\right]$ & $2 \mathrm{veh} / \mathrm{s}$ & $0.5 \mathrm{veh} / \mathrm{s}$ \\
\hline \multicolumn{3}{|c|}{ Applied to intersections } \\
\hline Intersection & Active phases & Green split $g_{p}^{j}(t)$ \\
\hline \multirow{2}{*}{5} & 1:West-East & 0.6 \\
\hline & 2:North-East & 0.4 \\
\hline \multirow{2}{*}{6} & $1:$ North-South & 0.4 \\
\hline & 2:West-South & 0.6 \\
\hline
\end{tabular}
run at the free-flow speed at the upstream of the link then adapt their speed according to the congestion downstream.

\section{A. Small Network}

Fig. 3 depicts the topology of the grid network in this case study. It consists of 11 nodes, 13 links, and a single origindestination pair $(\mathrm{R}, \mathrm{S})$. In this network, links 2_3, 4_5, and
TABLE IV

PARAMETER SETtings FOR GRID $3 \times 3$ Network TOPOLOGY

8_9 are defined as bottleneck links while the others excluding the virtual links (R_1 and 9_S) that dedicated for origin and destination are treated as normal links. The design of the phasing diagram for intersections is prescribed by the National Electrical Manufacturers Association (NEMA). And hence, there are two intersections considered with conflicted movements at nodes 5 and 6 . Note that, we ignored the intersection at node 9 due to the fact that link "9_S" is a virtual link for the destination. Regarding the green split $g_{p}^{j}(t)$, we assume they are constant over the studied time period. For the detailed settings of this network, we refer to Table IV.

1) Speed Harmonization: At first, we aim to investigate the harmonization of speed trajectories when vehicles travel along with the link. We used the Gini-index [34] to measure the homogeneity of speed profile where a smaller value of Gini-index means more synchronized speed and a higher value presents greater speed deviation. To this end, Fig. 4 reveals the histogram of the values of Gini-index at a series of specific links between SH and non-SH control scheme. It shows that $\mathrm{SH}$ vehicles result in much lower Gini-index values in the comparison with the original solution (without applying $\mathrm{SH}$ ), 


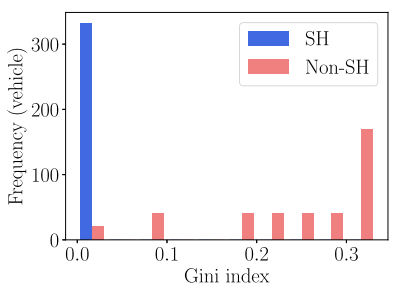

(a) Link 2_5

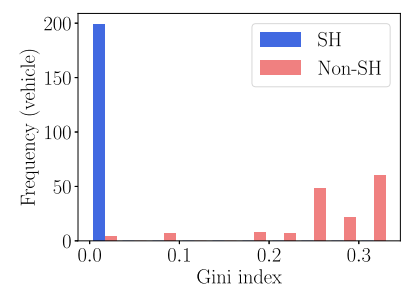

(b) Link 4_5

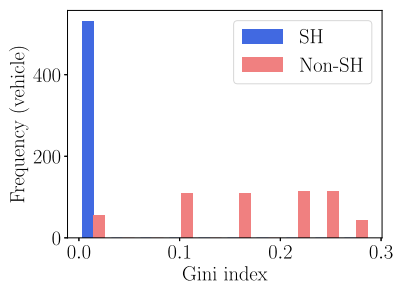

(c) Link 5_6

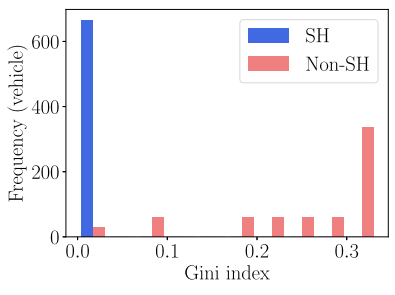

(d) Link 6_9

Fig. 4. Plots of histogram of speed homogeneity on individual vehicles within links between with and without SH where $\mathrm{x}$-axis is 'Gini index', $\mathrm{y}$-axis is 'Frequency (veh)' and the color represents the control scheme.

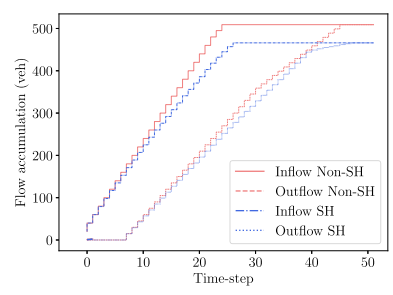

(a) Link 1_2

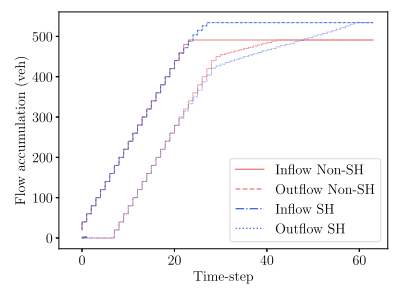

(b) Link 1_4

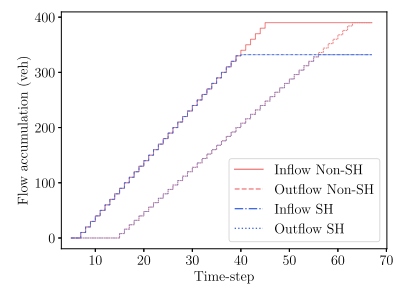

(c) Link 2_5

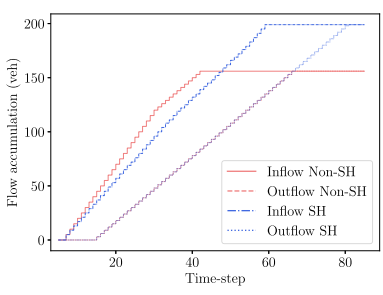

(d) Link 4_5

Fig. 5. Plots of traffic flow accumulation between with and without SH where x-axis is 'Time step', y-axis is 'Flow accumulation (veh)' and the color represents the control scheme.

which implies the effectiveness of the proposed framework in minimizing the variation of speed trajectories along vehicles' trajectories.

2) Flow Swapping: In the aforementioned formulation, it is observable that the maximum flow rate upstream of every link would be changed due to a speed advisory control scheme. Therefore, given the feedback of this variable to the upper level at every solving iteration, we found a significant change of traffic flow patterns in the studied network when the solution converged. This phenomenon is depicted in Fig. 5 for several diverging links where the traffic flow swapping takes place. It is worth noticing that the macroscopic model took a vital role in the queue dissipation process by positively balancing the traffic flow at all possible patterns throughout the constructed network.

3) Queue Formation: As a result of speed harmonization and flow swapping phenomena, the queue formation within individual links was significantly affected. The space-time density diagrams and space-time trajectory diagrams are shown in Figs. 7 and 6 to observe the formation of the queue within the link and the dissipation of queue inside the transportation network. In fact, vehicles with $\mathrm{SH}$ tend to run with a constant speed, or slightly varies around a constant speed, from entering to leaving the link, which explains the dense density at the beginning of the link. In contrast, non-SH vehicles prefer roaming with maximum permitted speed as long as they could until the congestion forcibly slows them down. Consequently, the density of non-SH is much higher at the end of the link, which could potentially lead to traffic breakdown before approaching the bottleneck link.

4) The Comparison of Network-Wide SH and Local SH: We measured the average speed of vehicles when they travel along with the link under a local SH and a network-wise SH control scheme. The comparison of this measurement for links that connecting to intersection 5 and 6 is shown in Fig. 8. As can be seen, network-wide $\mathrm{SH}$ improved the speed of vehicles by approximately $5 \%$ when they approach/leave the intersections. Besides, network-wide $\mathrm{SH}$ is also more effective than local $\mathrm{SH}$ in terms of providing speed homogeneity over the network with $14 \%$ less in speed variance that resulted from the potential impact on traffic flow patterns as shown in Fig. 5.

\section{B. Large Network}

To further evaluate the system-wide effect of the vehicle speed harmonization in a larger network, we use the FortWorth transportation network as shown in Fig. 9. This network consists of about 100 nodes and 400 links with 10 origindestination pairs where any link is assumed to have at least two lanes. For this network, we aim to estimate the complexity of the framework, investigate the network-wide speed harmonization in a more realistic traffic scenario and evaluate the environmental benefits of $\mathrm{SH}$ regarding fuel consumption and emissions of vehicles.

1) Estimation of Complexity: We consider the complexity of the model in three aspects regarding the number of constraints, the number of variables and the computational time. To this end, we also proposed three different configurations under the Fort-Worth network with different time step periods and demand profiles for the investigation as described in Table V. The proposed measurements are applied on all configurations mentioned in Table $\mathrm{V}$ and showed in Table VI. Note that, the data in Table VI is measured in terms of a single iteration for the macroscopic model and a single link for the microscopic model.

The complexity of the model comes from the macroscopic model, while the microscopic model is much more simple. It 


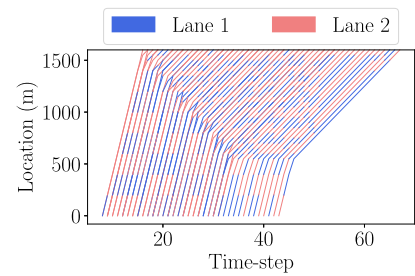

(a) Link 4_5 without $\mathrm{SH}$

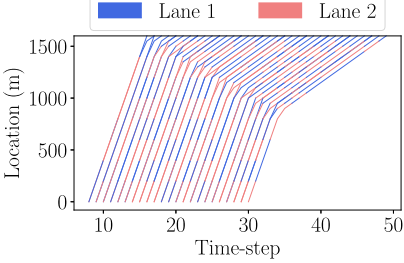

(b) Link 4_7 without SH

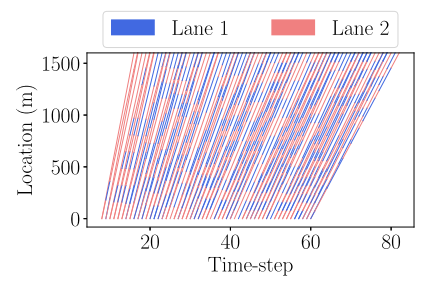

(c) Link 4_5 with SH

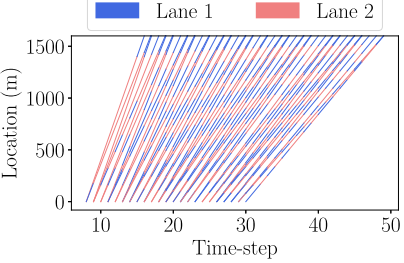

(d) Link 4_7 with SH

Fig. 6. Plots of space-time trajectory between with and without SH where x-axis is 'Time step', y-axis is 'Location (m)' and the colors (blue and red) represent the specific lane on which the vehicles are traveling, i.e. a color change within the trajectory indicates a lane change of that vehicle group.

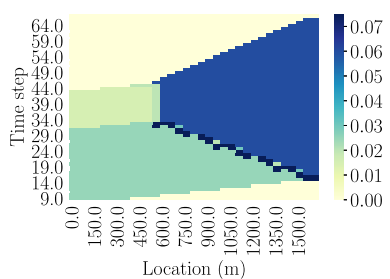

(a) Link 4_5 without SH

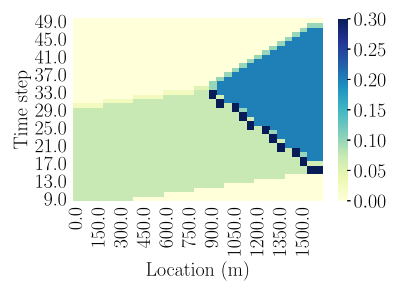

(b) Link 4_7 without SH

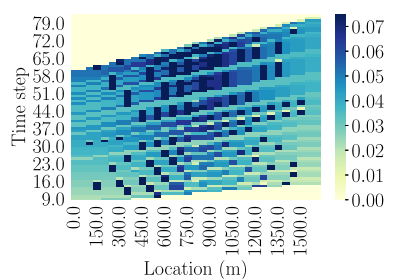

(c) Link 4_5 with SH

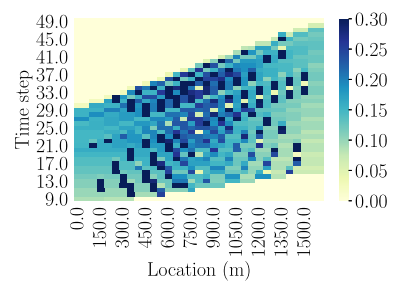

(d) Link 4_7 with SH

Fig. 7. Plots of space-time density between with and without $\mathrm{SH}$ where $\mathrm{x}$-axis is 'Location (m)', y-axis is 'Time step' and the color bar represents the density in the range $\left(0, K_{a}\right)(\mathrm{veh} / \mathrm{m})$.

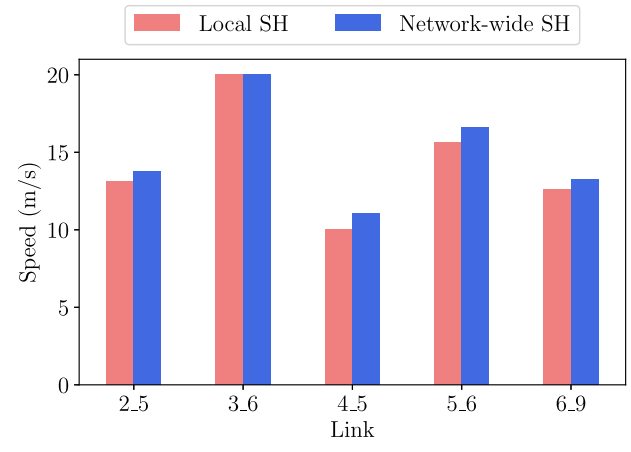

Fig. 8. Comparison of vehicles' average speed between local SH and network-wide SH control scheme on links connected to intersections of the simple network.

TABLE V

The CONFIgurations OF Fort-WORTH NETWORK

\begin{tabular}{cccc}
\hline & \multirow{2}{*}{$\begin{array}{c}\text { Time interval } \\
(\mathrm{min})\end{array}$} & \multicolumn{2}{c}{$\begin{array}{c}\text { Demand } \\
\text { Confeh/min) }\end{array}$} \\
\cline { 3 - 4 } & & R1-S1, R2-S2 & R3-S6, R4-S1, R4-S5, R5-S2 \\
& & R3-S1 & R5-S4, R6-S2, R6-S3 \\
\hline C1 & 2.5 & 6.25 & 0.75 \\
C2 & 2 & 12.5 & 1.25 \\
C3 & 1.5 & 25 & 2.5 \\
\hline
\end{tabular}

is also observable that as the configuration grows linearly in terms of a studied time step duration and demand, the number of constraints and variables in the macroscopic model grows exponentially. The detailed convergence profile of a large network case study is shown in Fig. 10 where the number of iterations satisfying the condition of convergence is from 8 to 9 iterations.

2) System-Wide Speed Harmonization Evaluation: In this section, we investigate the inter-links speed harmonization as an effect of the VSA-based algorithm. Hence, we calculated the space mean speed for links across the studied network

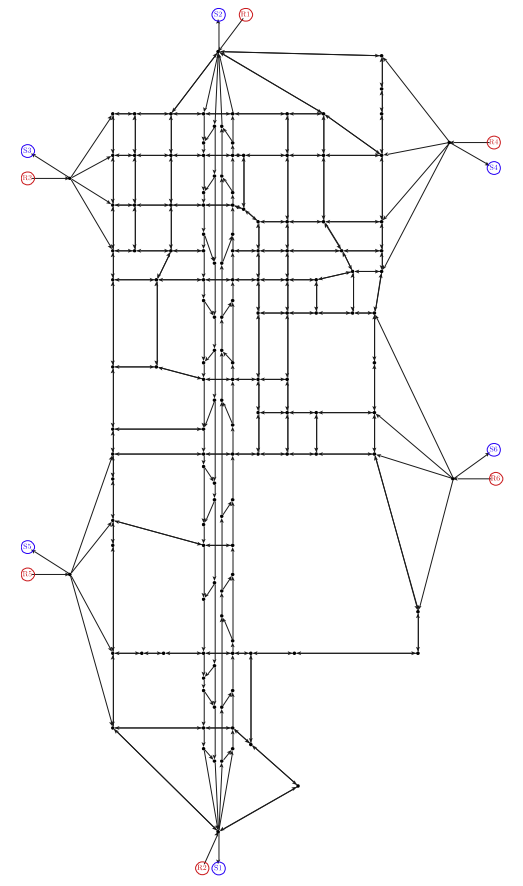

Fig. 9. Fort-Worth transportation network.

between non-SH and $\mathrm{SH}$ for the evaluation. It shows that the SH-based method produced a more uniform distribution of speed by reducing $8.3 \%$ in the speed variance and $9.2 \%$ in the Gini index compared to the original solution. Hence, this finding indicates that the implementation of vehicle speed harmonization or VSA-based algorithm can produce not only the harmonization regarding the speed trajectories of vehicles but also space mean speed of links over the entire network.

3) Fuel Consumption and Emissions: Although the optimization of fuel consumption and emissions are not directly formulated in our framework, it is expected that these metrics 


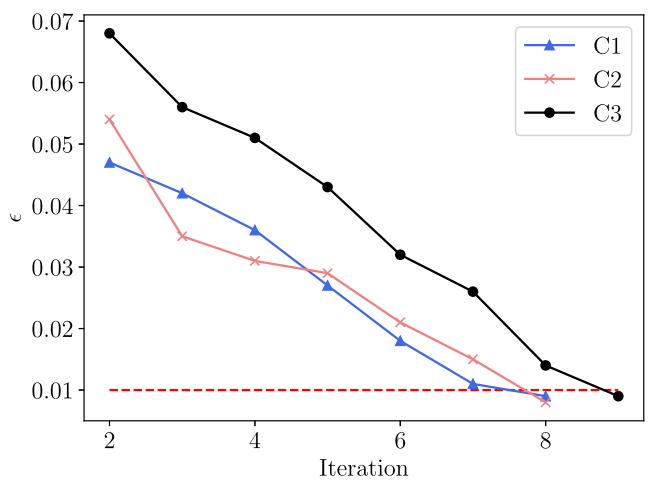

Fig. 10. Convergence performance of the large network case study.

TABLE VI

COMPleXity OF DifFerent Configurations

\begin{tabular}{cccccccc}
\hline \multirow{2}{*}{ Config. } & \multicolumn{2}{c}{ Constraints } & \multicolumn{2}{c}{ Variables } & \multicolumn{2}{c}{$\begin{array}{c}\text { Solving time (s) } \\
\text { per iteration }\end{array}$} & \multirow{2}{*}{$\begin{array}{c}\text { Convergence } \\
\text { (\#iterations) }\end{array}$} \\
\cline { 2 - 6 } & Upper & Lower & Upper & Lower & Upper & Lower & \\
\hline C1 & 439985 & 10098 & 676362 & 1512 & 3585 & 0.14 & 8 \\
C2 & 547809 & 21516 & 826664 & 3068 & 6836 & 0.45 & 8 \\
C3 & 795002 & 43341 & 1202419 & 6417 & 9690 & 0.82 & 9 \\
\hline
\end{tabular}

will be decreased due to small variations in speed profiles, which eventually prevents the unnecessary stop-and-go maneuvers. To this end, the VT-Micro model [35] was applied to estimate fuel consumption and different types of emissions such as Hydro Carbon (HC) and Nitrogen Oxides (NOx) to further exploit the potential benefits of SH to the environment. Note that, we utilized $\mathrm{C} 3$ configuration with the assumption that only a single-class user which is a light-duty vehicle type exists in the network. We calculated the aggregated amount of fuel consumption and emissions produced by vehicles under two different schemes regarding SH and non-SH control scheme. The result indicates the environmental benefits of $\mathrm{SH}$ by reducing $9.4 \%$ in fuel consumption, $8.5 \%$ in NOx emission, and $8.6 \%$ in $\mathrm{HC}$ emission.

\section{CONClusion}

In this article, we developed a novel bi-level formulation for a group-based dynamic speed advisory problem in a multiple origin-destination network. We proposed the macroscopic approach to minimize the total travel time of all CAVs in the network at the upper model while the speed profile of individual CAV is harmonized on each and every link in a group-based microscopic model at the lower level. To this end, we devised a novel method to translate the macroscopic link flows into the arrival, departure schedule, and turning direction of groups, as well as the outcome of the microscopic speed profiles into a constrained maximum flow rate of every link in the network.

In our numerical studies, we applied the proposed framework to a simple grid network as well as a Fort-Worth network to investigate the effect of speed harmonization and evaluate the performance of the whole network. The results showed that the total emission, fuel consumption, and queue formation within the network have been significantly improved with the proposed speed harmonization. Moreover, harmonizing the vehicle's speed within individual links further improved the uniformity of speed over the network, i.e., smaller change of speed from one link to another leading to better queue formulation and reduction in the vehicle's emission and fuel consumption. Network-wide SH control strategy is also more effective than a local $\mathrm{SH}$ control scheme regarding the improvement of the average speed of vehicles approach/leave intersections and the homogeneity of speed across the network.

\section{REFERENCES}

[1] Traffic and Congestion Cost Trends for Australian Capital Cities, Bur. Infrastruct. Transp. Regional Econ., Canberra, ACT, Australia, 2015.

[2] C.-Y. Yan, R. Jiang, Z.-Y. Gao, and H. Shao, "Effect of speed limits in degradable transport networks," Transp. Res. C, Emerg. Technol., vol. 56, pp. 94-119, Jul. 2015.

[3] Y. Yang, H. Lu, Y. Yin, and H. Yang, "Optimization of variable speed limits for efficient, safe, and sustainable mobility," Transp. Res. Rec., J. Transp. Res. Board, vol. 2333, no. 1, pp. 37-45, Jan. 2013.

[4] H. Yang, X. Wang, and Y. Yin, "The impact of speed limits on traffic equilibrium and system performance in networks," Transp. Res. B, Methodol., vol. 46, no. 10, pp. 1295-1307, Dec. 2012.

[5] M. Robinson et al., "Examples of variable speed limit applications: Speed management workshop," United States Dept. Transp., Washington, DC, USA, Tech. Rep. 2243, 2000.

[6] E. Kwon, C. Park, D. Lau, and B. Kary, "Minnesota variable speed limit system: Adaptive mitigation of shock waves for safety and efficiency of traffic flows," Transp. Res. Board, Washington, DC, USA, Tech. Rep. 11-3941, 2011.

[7] A. Hegyi, B. DeSchutter, and J. Hellendoorn, "Optimal coordination of variable speed limits to suppress shock waves," IEEE Trans. Intell. Transp. Syst., vol. 6, no. 1, pp. 102-112, Mar. 2005.

[8] A. Hegyi, S. P. Hoogendoorn, M. Schreuder, H. Stoelhorst, and F. Viti, "SPECIALIST: A dynamic speed limit control algorithm based on shock wave theory," in Proc. 11th Int. IEEE Conf. Intell. Transp. Syst., Oct. 2008, pp. 827-832.

[9] R. C. Carlson, I. Papamichail, M. Papageorgiou, and A. Messmer, "Optimal mainstream traffic flow control of large-scale motorway networks,' Transp. Res. C, Emerg. Technol., vol. 18, no. 2, pp. 193-212, Apr. 2010.

[10] F. Zhu and S. V. Ukkusuri, "Accounting for dynamic speed limit control in a stochastic traffic environment: A reinforcement learning approach," Transp. Res. C, Emerg. Technol., vol. 41, pp. 30-47, Apr. 2014.

[11] M. Tajalli and A. Hajbabaie, "Dynamic speed harmonization in connected urban street networks," Comput.-Aided Civil Infrastruct. Eng., vol. 33, no. 6, pp. 510-523, Jun. 2018.

[12] K. Katsaros, R. Kernchen, M. Dianati, and D. Rieck, "Performance study of a green light optimized speed advisory (GLOSA) application using an integrated cooperative ITS simulation platform," in Proc. 7th Int. Wireless Commun. Mobile Comput. Conf., Jul. 2011, pp. 918-923.

[13] V. Nguyen, O. Tran Thi Kim, T. Nguyen Dang, S. Il Moon, and C. S. Hong, "An efficient and reliable green light optimal speed advisory system for autonomous cars," in Proc. 18th Asia-Pacific Netw. Operations Manage. Symp. (APNOMS), Oct. 2016, pp. 1-4.

[14] X. He, H. X. Liu, and X. Liu, "Optimal vehicle speed trajectory on a signalized arterial with consideration of queue," Transp. Res. C, Emerg. Technol., vol. 61, pp. 106-120, Dec. 2015.

[15] S. Stebbins, M. Hickman, J. Kim, and H. L. Vu, "Characterising green light optimal speed advisory trajectories for platoon-based optimisation," Transp. Res. C, Emerg. Technol., vol. 82, pp. 43-62, Sep. 2017.

[16] R. K. Kamalanathsharma and H. A. Rakha, "Multi-stage dynamic programming algorithm for eco-speed control at traffic signalized intersections," in Proc. 16th Int. IEEE Conf. Intell. Transp. Syst. (ITSC ), Oct. 2013, pp. 2094-2099.

[17] J. Li, M. Dridi, and A. El-Moudni, "Multi-vehicles green light optimal speed advisory based on the augmented lagrangian genetic algorithm," in Proc. 17th Int. IEEE Conf. Intell. Transp. Syst. (ITSC), Oct. 2014, pp. 2434-2439.

[18] H. Yang, H. Rakha, and M. Venkat Ala, "Eco-cooperative adaptive cruise control at signalized intersections considering queue effects," IEEE Trans. Intell. Transp. Syst., vol. 18, no. 6, pp. 1575-1585, Jun. 2017.

[19] H. Jiang, J. Hu, S. An, M. Wang, and B. B. Park, "Eco approaching at an isolated signalized intersection under partially connected and automated vehicles environment," Transp. Res. C, Emerg. Technol., vol. 79, pp. 290-307, Jun. 2017.

[20] F. Zhou, X. Li, and J. Ma, "Parsimonious shooting heuristic for trajectory design of connected automated traffic Part I: Theoretical analysis with generalized time geography," Transp. Res. B, Methodol., vol. 95, pp. 394-420, Jan. 2017. 
[21] J. Ma, X. Li, F. Zhou, J. Hu, and B. B. Park, "Parsimonious shooting heuristic for trajectory design of connected automated traffic Part II: Computational issues and optimization," Transp. Res. B, Methodol., vol. 95, pp. 421-441, Jan. 2017.

[22] Y. Guo, J. Ma, C. Xiong, X. Li, F. Zhou, and W. Hao, "Joint optimization of vehicle trajectories and intersection controllers with connected automated vehicles: Combined dynamic programming and shooting heuristic approach," Transp. Res. C, Emerg. Technol., vol. 98, pp. 54-72, Jan. 2019.

[23] Y. Wei et al., "Dynamic programming-based multi-vehicle longitudinal trajectory optimization with simplified car following models," Transp. Res. B, Methodol., vol. 106, pp. 102-129, Dec. 2017.

[24] A. A. Malikopoulos, S. Hong, B. B. Park, J. Lee, and S. Ryu, "Optimal control for speed harmonization of automated vehicles," IEEE Trans. Intell. Transp. Syst., vol. 20, no. 7, pp. 2405-2417, Jul. 2018.

[25] I. Yperman, "The link transmission model for dynamic network loading," Ph.D. dissertation, Dept. Civil Eng., Katholieke Univ., Leuven, Belgium, 2007.

[26] S. Ukkusuri, K. Doan, and H. M. A. Aziz, "A bi-level formulation for the combined dynamic equilibrium based traffic signal control," Procedia Social Behav. Sci., vol. 80, pp. 729-752, Jun. 2013.

[27] W. Shen and H. M. Zhang, "What do different traffic flow models mean for system-optimal dynamic traffic assignment in a Many-to-One network?" Transp. Res. Rec., J. Transp. Res. Board, vol. 2088, no. 1, pp. 157-166, Jan. 2008.

[28] G. F. Newell, "A simplified car-following theory: A lower order model," Transp. Res. B, Methodol., vol. 36, no. 3, pp. 195-205, Mar. 2002.

[29] P. Hansen, B. Jaumard, and G. Savard, "New Branch-and-Bound rules for linear bilevel programming," SIAM J. Scientific Stat. Comput., vol. 13, no. 5, pp. 1194-1217, Sep. 1992.

[30] Gurobi Optimization. (2019). Gurobi Optimizer Reference Manual. [Online]. Available: http://www.gurobi.com

[31] T. Islam, H. L. Vu, M. Panda, and D. Ngoduy, "A study of realistic dynamic traffic assignment with signal control, time-scale, and emission," J. Intell. Transp. Syst., vol. 22, no. 5, pp. 446-461, 2018.

[32] D. Ngoduy, N. H. Hoang, H. L. Vu, and D. Watling, "Optimal queue placement in dynamic system optimum solutions for single origin-destination traffic networks," Transp. Res. B, Methodol., vol. 92, pp. 148-169, Oct. 2016.

[33] H. Bar-Gera, D. Boyce, and Y. Nie, "User-equilibrium route flows and the condition of proportionality," Transp. Res. B, Methodol., vol. 46, no. 3, pp. 440-462, Mar. 2012.

[34] A. Sen et al., On Economic Inequality. London, U.K.: Oxford Univ. Press, 1997.

[35] H. Rakha, K. Ahn, and A. Trani, "Development of VT-micro model for estimating hot stabilized light duty vehicle and truck emissions," Transp. Res. D, Transp. Environ., vol. 9, no. 1, pp. 49-74, Jan. 2004.

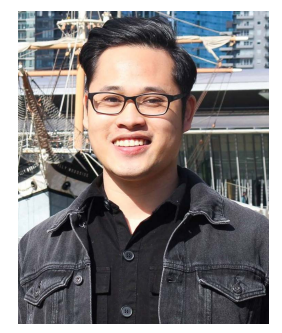

Cuong H. P. Nguyen received the B.Sc. degree in electrical engineering from International University, Vietnam National University, in 2016. He is currently pursuing the Ph.D. degree with the Monash Institute of Transport Studies, Australia. His current research interests include traffic control and management in transportation networks considering connected and autonomous vehicles.

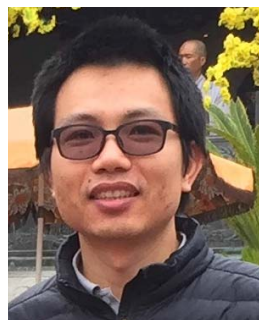

Nam H. Hoang received the B.Sc. degree in computer science and engineering from the Ho Chi Minh City University of Technology (HCMUT), Vietnam, in 2007 , the M.Sc. degree in telecommunication and computer networks engineering from the Politecnico di Torino, Italy, in 2012, and the Ph.D. degree from the Swinburne University of Technology, Australia, in 2017. Since 2018, he has been a Research Fellow in intelligent transport systems with the Engineering Faculty, Monash University. His research interest includes modeling and optimization in transport systems.

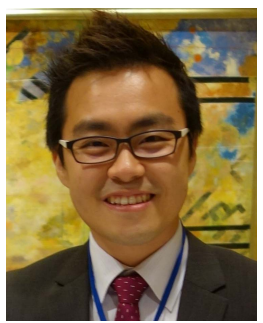

Seunghyeon Lee received the B.S. degree in transportation system engineering and the M.S. degree in transportation engineering from Ajou University, South Korea, and the Ph.D. degree from The University of Hong Kong. After two years of postdoctoral research experience at the University of Canterbury, New Zealand, he is currently a Lecturer with the Faculty of Engineering and Information Technology, University of Technology Sydney, Australia. His research interests include adaptive traffic control systems, stochastic traffic flow theories, and deep learning methods in transport modeling.

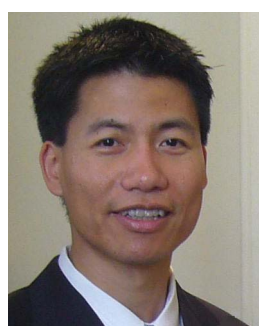

Hai L. Vu received the B.Sc., M.Sc., and Ph.D degrees in electrical engineering from the Technical University of Budapest, Hungary, in 1994 and 1999, respectively. He is currently a Professor of intelligent transport systems (ITS) with the Faculty of Engineering, Monash Institute of Transport Studies, Monash University, Australia. He is a leading expert with 20 years of experience who has authored or coauthored more than 180 scientific journals and conference articles in data and transportation network modeling, V2X communications, and connected autonomous vehicles (CAVs). His research interests include modeling, performance analysis and design of complex networks, stochastic optimization, and control with applications to connected autonomous vehicles and intelligent transportation.

Dr. Vu was a recipient of the 2012 Australian Research Council (ARC) Future Fellowship and the Victoria Fellowship Award for his research and leadership in ITS. 